\title{
Johann Laski und der Abenteurer Heraklid Basilikus.
}

\author{
Von Th. Wotschke.
}

Nach einem vierzehntägigen Aufenthalte in Warschan, wo Vergerio am 21. Jannar $1557 \mathrm{karz}$ nach Scblaß des Reichstages and nach der Abreise des Königs nach Wilna eingetroffen war and inmitten der noch versammelten Landboten in seinem bekannten Eifer für die Reformation gewirkt hatte, war er nach Krakau aufgebrochen. Bis zam 23. Februar weilte er in der polnischen Königsstadt, um dann nach Posen sich zu wenden. In dem Hause des bekannten Johann Boner, dem er soeben die Lowitscher ${ }_{n}$ Formula fidei “ mit der ihr gegenubergestellten Württembergischen Konfession gewidmet hatte, mag er abgestiegen und mit seines Gastfreundes Onkel Johann Laski zusammengetroffen sein. Hier predigte ja dieser, soweit es seine schwache, darch die anstrengende Heimatfahrt im Spätherbst des vergangenen Jabres noch mehr geschwächte Gesundheit and daza seine Arbeit an der polnischen Bibelubersetznng des Orsatius gestattete. ln seinen Gesprächen mit Laski wird der ehemalige Bischof von Capodistria anch jenes griechischen Ritters und Abenteurers Jakob Heraklid Basilikns ${ }^{1}$ ) gedacht haben, den er Ende Juni 1556 in Melanchthons Hause getroffen, der in diesem Monat darch die Dichterkrönung zweier jungen Nenlateiner sich einen Namen an der Leukorea gemacht ${ }^{2}$ ), der ihn vielleicht auf seiner Reise von Wittenberg nach Mecklen-

1) Über ihn vgl. Wotschke, Kirchengeschichtliches rom ramïnischen Kriegsschauplatze. Theolog. Literaturbericht 1917, S. 29-34.

2) Vgl. Wotschke a. a. O. S. 29. Das griechische Zeognia, das Melanchthon im Namen des Heraklid dem jungen Dichter Zacharias Prätorius susstellte, findet sich Corp. Ref. VIII S. 837 Nr. 6006. 
burg begleitet hatte, und der ihm dann im folgenden November nach seiner Ruckkehr ans Wilna in Königsberg wiederbegegnet war. Wabte er doch, daB dieser Abenteurer soeben auf die Kunde von dem Aufbrache des Königs ron Warschan nach Wilna nach Lithauens Hatuptstadt geeilt war, am 23. Januar sich vom Herzoge. Albrecht an den polnischen Herrscher hatte empfehlen lassen. Laski, der selbst zur Reise an den könig. lichen Hof sich anschickte, mobte ihn dort treffen, kannte ibn gewiB auch schon, zam mindesten aus den Berichten anderer, etwa des Grafen Volrad von Mansfeld, unter dem der griechische Ritter gedient batte.

In der Tat sehen wir den polnischen Reformator, der an 17. März in Wilna eintraf ${ }^{1}$ ) und hier einen Monat beim Fursten Nikolaus Radziwill ehrenvolle Gastfreuudscbaft genoB, bald in enger Verbindung mit Heraklid. Das Bekenntuis zur Reformation, die gemeinsame Verehrung des Herzogs Albrecht, die gemeinsame Wertschätzang Melanchthons fuhrte sie and etliche andere wie Johann Trzecieski, den namhaften Humanisten, Stalislaus Kossacki ${ }^{2}$ ), den königlichen Bibliothekar und Übersetzer der Erziehungslehre des Marburger Lorichins, Johann Maczinski ${ }^{8}$ ), den Radziwillschen Geheimsekretär, zusammen. Ein junger Wilnaer Theologe Cyprian, der in Heraklids Dienste trat, anf seine Fursprache vom Könige damals den Adelsbrief erhielt, sich seitdem nach seinem Gönner Heraklid Basilikus nannte, empfing ans Laskis oder seines Begleiters Utenhure Hand den ersten Teil der bekannten Geschichte der Londoner Fremdengemeinde, die Utenhove im nächsten Jahre nach der Schweiz schickte, wo sie Oporin in Basel endlich März 1560 drackte ${ }^{4}$ ). Er hat sie später ins Polnische übersetzt und seinem Märtyrerbuche

1) Über Laskis Reise ron Krakau n้ach Wilua rgl. Kunheinis Briefe vom 26. Februar und 21. Närz 1557 an Herzog Albrecht bei Wotschke, Abraham Culvensis. Altpr. Honatsschrift XLII, S. $205 \mathrm{f}$.

2) Vgl. Wotschke, Erasmus Glitzner. Jahrbnch d. Vereins f. Kirchengesch. Posens 1918, S. 6.

3) Vgl. Wotschke, Vergerios zweite Reise nach Preußen und Polen. Altpr. Mouatssehrift 1911, S. 252 f.

4) Calvin lehnte es ab, die Vorrede zu schreiben, der $\cdots \cdots$ ter Drucker Crispin, das Buch zu veröffentlichen, Kalisch, den 26. Mërz 1558 schrieb darauf Laski die Vorredo. 
beigegehen '). Da (yprian musikalisch interessicrt war²), mağ er auch den königlichen Kapellmeister Bakfark aus Ingarn, der gleichfalls Beziehungen zu Herzog Albrecht hatte. und seinen Schwager Lorenz Kryszkowski, den späteren Lnitarier ${ }^{y}$ ), in den Kreis um Laski and Heraklid gezogev hashen. Auch Erhard ron Kunheim, der Brader jenes Georg Kunheim, der Lathers Tochter Margarete heingefuhrt bat, Hofmeister der Königin, von Herzog Albrecht beanftragt, Heraklids Anliegen beim Könige za unterstützen, mag ibm nicht ferugestanden haben. Interessant ist das Schreiben. das der griechische Abenteurer dem Herzoge sandte ${ }^{4}$ ):

1) Vyl. Wotschke, Gescuichte der Reformation in Polen. S. 2tit.

2) Vgl. sein Schreiben an Herzog tilbrecht, ds er ihm eiue nusiE... ocle Komposition schickte: ${ }_{n} A b$ infantia usqne mea semper mihi hoc erat curae, ut in summoram virorum, eornu potissimum, quos bomines suspiciunt omnes, gratiam derenire possin boc ipsum collocans magnae felicitatis loco, si in principam clientela vivens eornm patrocinio at gratia non contemnenda ganderem. Et cum andirem Ill. Celsnis $\nabla$. heroicss virtutes et opera magnifica, quorum fams totum ferme penetravit orbem, optavi, ut saltem liceret mannm Ill. Celsnis $\nabla$. cum humillima servitiorım meorum conmendatione exosculari. Illa etiam Ill. Celsnis V. vere christiano principe digna pietate atque adeo erga omnes studiosos gratis singulari fretus induri in animum Ill. Celyni $V$., domino meo clementisaimo, aliquid laboris mei dedicare, at eo ipso gratiam Ill. Celsnis V. mihi conciliare et in numerum clientum Ill. Celgnis V. perrenire possim. Etai antem haias rei mihi conscius sim, hoc munusculum meum tanto principe minus dignum esse, credo enim Ill. Celsnem $\nabla$. longe meliora ab excellentissimis nostrae aetatis musicis habere, nihilominus tamen gratiae Ill. Celgnis $\nabla$. fidens spero IIl. Celgnen $\nabla$. tam benignis auribus velle andire corvi strepitum atque cygni dulcissimam cantum, quandoquidem cygno canente liberum est corvo crocitare. Quapropter obuixe peto, illustrissime princeps, ut hoc parvum munusculum a me oblatum placido vultu excipere meque in sua gratia atque patrocinio clementer conservare dignetar. Dominus deus Ill. Celsnem V. din nobis servet incolumem ad reipublicae christianae utilitatem et ecclesiae suae consolationem. Dat. Vilnae 29. Novembris anuo salutis 1560. Ill. Celsnis V. humillimus servitor Cyprianus Heraclides Basilicus.

ง) Über Bakfark und Kryszkowski vgl. Wotschke, Vergerios zweite Reise nach Preußon und Litauen. Altpr. Monatsschrift 1911, S. $266 \mathrm{ff}$.

4) Das Schreiben ist wie sämtliche andere Urkunden dem Staatsarchiv in Königsberg entnommen.

Archip far Reformationegerobichte. XVII. 1. 
Illustrissine princeps, domine clementissime. Quod saperioribus diebus V. C. nullas scripserim literas, cansae, quod nullum adhac a regia maiestate acceperim responsum, ascribere velitis, non meae negligentiae. Nolui itaque intermittere, quin oblata occasione $V$. C. de statu onnium rerum mearum, quarum V. C. solam fuisse promotorem scio, certiorem redderem, imprimis vero pro omnibus beneficiis a V. C. mihi exhibitis similiter pro honesta commendatione gratias quanı maximas habeo, et si non satisfacere possum, ad minimum pro beneficiis cognoscere possum ac potero. Regiae maiestati servitiis sum obstrictus et devinctus, inprimis vero donavit ne 300 taleris, equum quoque pro mea persona pollicitus est. Insuper promisit se cum Turca de restitutione meae patriae acturnm, quo mea dulcissima patria potiri possim, et propter hanc solam causam se missuram proprium legatum ad Turcam. Praeterea offendi hic legatum patriarchae Constantinopolitani, cuins frater est episcopus sami et agnatus Pari, cui totum negotium commisi. Non dubito farente divina clementia me patriam iterum habituram et recepturum. Huius tantae spei et solatii principalem causam faisse V. C. agnoseo. Ab illustrissimo principe palatino V'ilnensi multis beneficiis sum affectus ac in dies alficior, qui imprimis ex singulari sua pietate et impulsione marischalchi reginae, ad quem V. C. scripsit meo nomine, mea negotia apud s. r. maiestatem tractavit. Rogo itaque V. C. etiam atque etiam, me apad ill. principem palatinum Vilnensem literis data occasione aliqua commendare meque in numero vestro. rum ministroram ac intimoram retinere dignetur, ut saa celsitudn videat $V$. C. esse adhac memorem sui servi.

Fuit et hic, illustrissime princeps, domive clementissime, diebus superioribus dominus a Lasco, bomo multae et admirandae eruditionis, virtatis ac pietatis, qui abique pietatem et alias virtutes V. C. occasione aliqua oblata praedicabat, imo etiam gloriabatur de multis et magnis donis a V. C. sibi exhibitis. Cum tam bene affectum illum erga V. C. viderem, interrogavi, atrum nobiseum sentiret de iustificatione. Ille publice multis praesentibus et andientibus, qui nos haereseos damnant, dixit se nos omnes et Osiandram habere pro fratribus in Christo neque illum suo suffragio ab ecelesia expellere, imo omnes dicebat, qui vos et Osiandrum hereseos damnant, pessime facere et horribiliter peccare. „Quicquid enim", inquit, "Osiander scripsit, eins habuit legitimam causam, et qui scribuut contra illum et tanquam haereticum damnant, male faciunt." „Sed hoc", inquit, „possunt dicere et scribere in usarpatione vocabaloram fuisse errorem, sed non haeresim ${ }^{4}$. Quod mihi valde placuit itaque haec V.C. scribere volui. Utinam atque ntinam aliquando daretur 
occasio, at a V. C. rocaretor ${ }^{1}$ ), ut sua authoritate et doctrina illos nostros adrersarios confunderet. Quantum de sacramento attinet, dixit mibi et omuino voluit, at etiam V. C. scriberem se libenter velle convenire cum omnibus et rem in terminos tales constituere, ut omnes conveniamus, ne, inquit, sit divisio inter evangelicos in gaudium et exultationem papistarum et scandalum priorum. Non landavit Philippum Melancthonem, quod ita in prima epistola Pauli invehatar in Osiandram. Vilnae 23. die Aprilis. Vrae Cels ${ }^{\text {nis }}$ deditissimus servus Basilicus despotas.

Als Heraklid diesen Brief schrieb, war Laski bereits aufgebrochen, um ther GroBpolen nach Krakan zortlckzakehren. Hierher lenkte auch Heraklid nach karzer Teilnahme an dem kampf- and rahmlosen Kriegszage gegen den Ordensmeister Furstenberg seine Schritte. Sigismund Angust hatte ihm Empfehlnugsbriefe an den Sultan mitgegeben, der Abenteurer scheint anfänglich also nach Konstantinopel sich haben wenden za wollen, aber Laski und seine Freande Stanislans Lasocki nod Hieronymas Philippowski, die Fubrer des nnternehmungslustigen kleinpolnischen evangelischen Adels, hielten ihn fest. Er hatte zar reformierten Abendmahlslehre sich bekannt ond seine Verwandtschaft mit dem Hospodar der Moldau Alexander "entdeckt", der mit ihm am Feldzag gegen Livland teilgenommen. Konnte er nicht vielleicht an Alexanders Stelle zum Herrn der Moldau sich aufschwingen and dieses Land der Reformation erschließen? Mit Herıog Albrecht blieh er im Briefwechsel, sandte ihm auch sein Buch uber kriegstechnische Fragen, das er im Anschlaß an die Livlandfahrt, vielleicht unter Benutzong des Kriegsbuches des Hieronymus Laski, geschrieben und das Trzecieski mit Versen begrußt hatte. Als der Hohenzoller ihm am 28. Oktober 1557 dankte $^{2}$ ), ihm sein Bild

ग) 1554 lief das Gerücht am, Herzog Albrecht würde Laski zam Bischof berufen. Wotschke, Herzog Albrechts Briefe an Laski. Altpr. Monatsschrift S. 45, 459.

7) „Serenissime domine. Binas literas a Dominatione Tua superioribus his diebus accepimus, alterae Horatium Curionem commendabant, alterae vero de disciplina militari librum sibi habebant coniunctum. Utraeque non dubias Domnis T. erga nos veri amoris continebant significationes, propter quas nobis etiam erant gratiores. Horatium autem, tum ut Domis $T$. consiliis, qnae amplectebamur, 
und eine Geldunterstiutzung schickte, gab er dem Boten ein Schreiben an Laski mit. Er war über dessen Aufenthalt

fieret satis, tum quia ex minerva eins elncebat coniuncta cum prudentia bonitas et pietas, nobis adscivimus. Reditum in sua Domni T. non invidemus praecamurque deum Domnis $T$. coepta consiliaque feliciter promoveat provehatque. Sed molestius id ferimus, quod multa adhuc reliqua habeamus, de quibus cum Domne T. conferre cupiebamus, et id quidem non tam eo quod militiae terrenae aucupemur gloriolam affectenusque, ut qui ad coelestem potius aspiremus, quam quod ei studio a pueritia dediti nobis ipsis nunquam faciamus satis. Et quod cupiamus commentationes nostras prodesse posteris aut certe nostris testatum faciamus, nos de disciplina militari veteri undique prementibus nunc hostibus non abiecisse curan studiumque, remittimus librum, ut, quae desideramus, suppleantur aciebusque numeri sui addantur, de quibus coram Horatius noster. Effigiem nostram, quam Domtio T. petiit, mittimus et viaticum, tenuius id quidem et opinione sua et nostra volnntate, sed quid faciamus? Tantum non exhausti et quantos hoc tempore fecerimus sumptus, Dom tio T. facile aestimare potest. Hoc solo Dom ${ }^{\text {ni }} T$. satisfaciemus, quod vestri memoriam nunquam sumus desopituri. Contra postulamus de suis rebus et Turcicis frequentes ad nos mittat literas. Valeat Dom tio T. quam felicius. Konispergae 28. Octobris a 1557."

Heraklid antwortete: "Ill. Celsnis V. literas accepi cum effigie ac viatico, pro quibus infinitas gratias ago. Mihi enim ista a Celsne V. gratissima sunt sciens animi sui erga me propensionem, et cum mihi nunc alius modus non sit gratiarum agendarum, humanitatem, benevolentiam, liberalitatem suam ac infinita in me beneficia praedicare ubique non cessabo. Quod d. Horatiom in suum servitium acceperit, non mediocriter gaudeo non solum illius causa, quod tanti prineipis servitium assecutus sit, sed etiam Ill. Celsnis V., quod sciam illam habituram servum industriam, fidelem atque diligentem et, quo magis eo utetur, sibi gratiorem. Si mihi experientia notus non fuisset nec existimassem eum idoneum futurum, profecto nunquam nec ipsum nec alium quemvis commendassem. Quare rogo Celsnem V. Ill., gratiosam illi se praebeat et non facile malevoli cuinsque voluntati credat. Is quoniam servos suos ac supellectilem hic non invenit, cogitur Schluzcii usque proficisci ad ducem Sclucensem. Ibi quamprimum fieri potuerit, se expediet ac ad Ill. Celsnem V. se conferet. Ego iam in ipso discessu sum sperans me brevi et patria et patrimonio potiturum, de quo gaudeo, quoniam erit mihi maior occasio demonstrandi benefactoribus ac patronibus (!) meis animi gratitudinem. Librum corrigam ac per d. Horatium mittam. Deus Ill. Celsnem V. cum ill. coniuge et filio ill. in per-

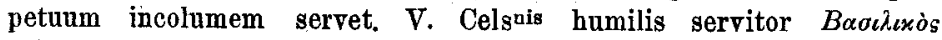

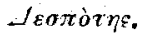


im unklaren ${ }^{1}$, scturieb deshalb auch am 30 . Oktober an dessen Neffen Nikolaus Laski, scheint ihn aber in Heraklids Niihe rermutet zo haben.

Laski arbeitete damals an eiuer Verschmelzung aller Erangelisehen Polens zu einer einigen polnischen Nationalkirche. Für diesen kirchenpolitischen Plan gewann er Heraklid. Da er nur boffen konute unter Zustimmang and mit Einwilligung des Herzogs Albrecht seinen Plan zu verwirklichen, bestimmte er Heraklid, seinen Einflub auf inn in dieser Richtang geltend za machen. Er veranlaßte ihn, litenbores Agende dem Herzog zu schicken, diesen und ibn selbst in zwei Schreiben den Herzog za empfeblen, als er im Verfolg sełner Absichten stch Neujahr 1558 zar Reise nach Königsberg eutschloB ${ }^{2}$ ).

Illastrissime princeps ... Saepe meis literis solitas sum multos commendare V. Cels ${ }^{\text {ni }}$, quod non impudentiae, sed hono animo adscribere dignetur, etiam atque etiam rogo. Scio enim et ipse V. Cels ${ }^{\text {nis }}$ pietatem expertus sum, de qua minime abutens sed atens soleo bonos et honestos, qui propter religionem patiantur persecutionem, commendare. Nam eos ita gratos V. Cels ${ }^{\text {ni }}$ esse scio, ut per beneficentiam restram re ipsa experiantur. Superioribus diebus cam d. Curione ad V. Cels ${ }^{\mathrm{nem}}$ miseram una cum meo ministro quandam formam precum ecclesiasticarnm a praesentium latore loanue Utenhovio descriptam nna cum testimonio per rev. d. Ioannem Alascho dato sni exilii. Cam iam ipsemet d. Utenbovius ana cum rev. in Christo patre et propheta dei d. Alaseo in aulam V. Cels ${ }^{\text {nis }}$ suam iter statuit, volui eum pancis V. Cels ${ }^{\mathrm{ui}}$ commendare, quamvis ea est pietate et virtute praeditas, ut nullius indigeat commendatione. Quandoquidem, ill. princeps, dictus d. Utenhovins ex equestris ordinis familia prognatus, singulari prudentia, eruditione, pietate et virtute praeditus est, adeo ut rev. d. Alasco, ne meum tantum iudicium adferam, non solum eum magnifaciat, verum etiam, ut ab ipsomet rev. d. Alaseo audivi, admiretur, cum

1) Vgl. Wotschke, Herzog Albrechts Brief an Laski. Altpr. Monatsschrift 45, S. 460.

2) Konin, den 23. März 1558 bittet Laski anch Melanchthon, für ihn an den Herzog za schreiben. Daß er in diesem Briefe seines neuen Frenndes, der vor zwei Jahren Helanchthons Wohlwollen erfahren, nicht gedenkt, kann nicht weiter auffallen. Er wird seit seiner Abreise alls Krakau ohne Nachrichten ron ihm gewesen sein. 
vero propter reram, sanctan et catholicam religionem exilium ') cum maxima reram penuria patiatar, rogo V. Cels ${ }^{\text {nom }}$ obnixe et ut unus ex fidelissimis V. Cels ${ }^{\text {nis }}$ servis peto et obsecro, si propter illum menm animam erga V. Cels ${ }^{n e m}$ fidelem aliquem $V$. Cels ${ }^{\text {nis }}$ habeam farorem, ut istum nobilem commendatum babere dignetur iavetque et suam humunitatem et liberalitatem, quam solita est omnibus extraneis ostendere, exhibeat, Nam praeterquam est deo gratum tantum virum nobilem propter Christam dominam exulem iuvare, tanta est virtus in homine, quod abilibet terraram doctissimis viris reformatoribusque passim ecclesiis, ubi faliniater (!) est notus, V. Cels ${ }^{\text {is }}$ pietatem, liberalitaten patefaciet et praedicabit. Ideo iterum atque iterum obnixe rogo, at re ipsa illam, quam ego pietatem, liberalitatem praedicari et praedico de V. Cels ${ }^{\circ \bullet}$, experiatur singularique amore ac favore suo prosequatur. Quantam rev. d. Alasci autoritas adversus maledicos iuvabit, melins, quam ego scribere possum, intelliget. Quanta bona praedicat et omnibus de V. Cels ${ }^{\text {ne }}$ sua rev. dominatio dicit, seribere non possum, nam ubique, et ipse propriis anribas audivi, non solum hamanitatem, pietatem, liberalitatem, sincerum erga Christum affectum V. Cels ${ }^{\text {nis }}$ dicit et praedicat, verum etiam cum maximo affectu animoque benevolo contra omnes maledicos, qui vel ex invidia vel insania aliquid egurgitant, se opponit defenditque. Quod certe signum est maximae amicitiae et res non levis momenti, tantas vir tantae autoritatis se malevolis, qua tamen digna est V. Cels ${ }^{\text {do }}$, opponere, plaraque ego audivi et interfui. Vestrae Cels ${ }^{\text {ni }}$ scriberem, visi scirem V. Cels ${ }^{\text {ni }}$ iam ante multos annos suam rev. dominationem notissimam esse. Quod residuum est, rogo deum, patrem domini nostri Jesu Christi, ut V. Celsuem Nestorios annos servet incolunem, at pii habeant aliquod refugium. Datae Cracoviae, die 2. Januarii 1558. V. Ill. Cels ${ }^{\text {nis }}$ fidelissimas servitor Basilicas despotas Sami.

Etsi non sum bonas scriba, tamen manu propria V. Cels ${ }^{\text {i }}$ Ill., at cognoscat, quo affectu commendem istum pium nobilem, scripsi.

Obwohl Heraklid schon in diesem Briefe Laski anfs wärmste dem Herzog empfoblen hatte, richtete er am folgenden Tage für ihn an den Fursten noch folgende Zeilen: „Illustrissime princeps et domine domine clementissime. Post debitam meorum servitioram oblationem Cels ${ }^{n+m}$ V. certiorem facere volui rev. in Christo patrem et prophetam dei, $d$. Johannem Alascum visitandi gratia V. Cels ${ }^{n e m}$ accedere. Quo-

1) Sommer 1559 kehrte Utenhove nach England znrück. Am '?. Juni dieses Jahres woilte er schon in Frankfurt a. M. 
niam vero, illastrissime princeps, hic dictus d. Alaseus, ultra quam quod est vir adniranda pietate, prudentia, eraditione et sincrulari virtate praeditus, tum et $V$. Cels ${ }^{m i}$ fidelissimus et integerrimus serritor et amicas, imo talis ut $V^{\text {ra }}$ Cels $^{\text {do }}$ vestri honoris et famae per totam Poloniam, Lithuaniam, taceo Prussiam et Germaniam, vix habeat stadiosiorem, caias rei ego ipsemet aliquando sum testis, nam saepissime Vilnae et in Polonia interfui, ubi Vrae Cels ${ }^{\text {nir }}$ aliquaudo ant per me aut alium quendam $V^{\text {rae }}$ Cels ${ }^{\text {is }}$ fidelem mentio est facta, tum si quidam malevoli suum venenum evomere sunt conati, tum sua rev. dominatio non solum rationibus scripturaram, sed etiam obiurgando pro suo solerti ingenio eos confundebat; multa alia ego observavi, quae me de singulari affectu erga $V^{\text {ram }}$ Cels $^{\text {nem }}$ deditissimam certificarant, ideo Vram Cels $^{\text {nem }}$ rogare volui, at dictum dominum reverendissimam singalari humanitate, liberalitate et amore prosequi dignetur. Nam quantum sua autoritate non solam verbis sed et scriptis sais poterit obesse calumniatoribus, Vra Cels $^{\text {do }}$ cogitet. Quapropter supplex rogo Vram Celsuem, illum tali bonore, quo nunquam principem rel comitem ant palatinum aliquem prosecuta est, prosequi dignetur et ita liberaliter dimittere, at nnquam aliquis a Vra Cels $^{\text {ne }}$ est dimissus, et, si fieri posset, annuo stipendio sibi dedicare, quod et $V^{\text {rae }}$ Ill. Cels ${ }^{\text {nis }}$ filio et atile et honorificam fore non diffido. Si $\mathrm{V}^{\mathrm{ra}}$ Cels ${ }^{\mathrm{do}}$ meis his precibus et consilio locum dederit, non poenitebit et aliquando pro eo mihi gratias aget. Cum sua rev. dominatione veniet quidam nobilis, d. Utenhovius, singularis virtutis et pietatis homo, magnae antoritatis apud rev. suam dominationem et omnes nostras ecclesias, qui quoque propter evangelium et confessionem exilium patitur, de cuius statu latius ${ }^{\text {rae }}$ Cels $^{\text {ni }}$ scribam. Rogo obnixe, Vra Cels ${ }^{\text {do }}$ velit esse clemens. Deus opt. max. Vram Celsnem Nestoreos in annos servet incolumem. Datae Cracoviae 3. Januarii 1558. Vrae Ill. Cels ${ }^{\text {nis }}$ servitor fidelis Basilicas, Despotas Sami manu propria.

Laski hat Heraklid nicht wiedergesehen. Als er von seiner Reise nach GroBpolen ond Preaßen zaruckkehrte, war der Abentenrer nach der Moldau za seinem angeblichen Verwandten, dem Hospodar Alexander, gegangen ${ }^{1}$ ) und als

1) Jassi, den 25. Mai 1558 bittet Heraklid den Herzog um Waffen: ${ }_{n}$ Confugio ad $\nabla$ ram Celsnem, ut nobis auxilio esse dignetur subministrans arma aliqua ad includendum sicuti loricas vel squameas vel alterius generis et aliquot pixides sen sclopetas cum binis levissimis et campestribus tormentis bellicis ex minimis, qus apud Vram Celsnem reperiuntur. Res enim nostrae in huinsmodi sunt stata constitutae, ut non absque aliquo moto bellico in patriam reverti sperem... Quidquid ad 
er hier seines Lebens nicht mehr sicher war, zam Woiwoden von Reußen, Prokopius Sieniawski ${ }^{1}$ ), and schließlich zum Neffen des Reformators, Albrecht Laski, dem sobne des Diplomaten Hieronymus Laski, dem berichtigten Kondottieri, vach Kesmark in Lngarn. Am 8. Januar 1560) starb Johamn Laski. Heraklid aber sammelte mit Lasockis, Pbilippowskis und Albrecht Laskis Hilfe Ende 1560 Trappen, fiel in die Moldau ein, schlug den Hospodar Alexander am 18. Nor. 1561 bei Werbia aufs Haupt and machte sich zum Fürsten der Walachen ${ }^{2}$ ). Jetzt zeigte sich, welchen Fifer fur die Reformation Laski and seine Freande in dem Herzen des griechischen Ritters geweckt hatten. Ungeachtet aller entgegenstehenden Scbwierigkeiten suchte er sie in dem eroberten Lande einzuführen. In diesem Bestreben lieb er auch nicht nach. als er mit Albrecht Laski zerfallen war und ihm das pfandweise iiberwiesene Chotin wieder abgenornmen hatte ${ }^{3}$ ).

me mittere Vrae Cels"i visum fuerit, rel per potrodas vel per navim usque ad sondomiriaun et exinde huc dirigantur in Moldariaru penes ill. d. Alexandrum, Joldaviae et Valachiae waiwodam. ${ }^{\star}$

1) Vgl. Wotschke, St. Lutomirski. Archiv f. Reformationsgesch. III, 168.

2) Zur Ergänzung der Nachrichten, die ich hierüber in Theologischen Literaturbericht gegeben habc, rerweise ich auf das schreiben, das Gabriel Tarlo Wilna, den 6. Januar 1562 dem Herzog Albrecht sandte: „Daß der Despota den Woywoden ans ter Wallachey Alexandrum vertrieben und das Lanlt mit List erohert und eingenuhmen, werden E. F. D. zweiffelson danou lengst bereidt Kuntschaff empfangen baben. Und hatt derohalben gemelter Woywoda itzt sein Bottschait za Lonsde, so woll auch der junge Wayda ans Siebenbïrgen, bei welchen er sich. wie man sagt, aufhalten solle. Was aber jre liewerbe, kamn man noch alhier nitt wissen, weil sie die Zeitt dieselben bey der kön. Ilajt noch nitt abgeleget, die Zeitt aber wird es geben. In Gerneiu will man es dafür achten, es werde der Despota scliwerlich das Regiment lang fürenn, dan sich der tïrkisch Kayser des vertriebenen Vaywoden ernstlichen annehmen rnd gentzlichen des Fürhabens sein solle, inen widerumb zn restituirenn." Der Beglanbigungsbrief, den Heraklids Kämmerer und Gesandter Lencze dem polnischen Könige überreichte, vom 12. Dezember 1561 datiert, wie auch seine Instruktion befinden sich im Königsberger Staatsarchiv.

3) Achill Scipio schreibt Lemberg, den 24. Januar 1563 dem Herzog Albrecht: ${ }_{n}$ Wie ich mith schwerer Krangheith in der Reyzchen Lembergk mein Krangheith gelegen, hath der walachische Woywoda 
Deu keinpolnischen Geistlichen Johann Lasenski, der in Zurich und Genf woblbekannt war, berief er zum evangelischen Bischof des Landes, den Ratschlägen des Laskischen Schwiegersohnes Stauislaus Lutomirski lieb er willig Obr, den bekannten Lismanino suchte er als Theologen zu gewinnen. Sein früher Sturz ${ }^{1}$ ) and $\operatorname{Tod}^{2}$ ) November 1563 aber als der Lysspodt das sohlob Chodtin dem Lasko mith Gwalt als mith funttitasend llan abgedrungen und eingenomen, welch Slob der walachische Woywoda dem Lasko zu einem Pfandschillingk ror geinen darhgesezten Unkosten eingegeben, welchs achte ungeferlich vor Agnetis gescheen, und das feste HaıB so in der Walachay leith zwo Meylen ron Kamenezen am Nester gelegen. Waß nhue ferner daraus erfolgen wiell, wirth die Czeith geben. Omne regnum in se divisum dissolabitur." Über Aclill Scipio Schellenschmidt, der eine Chronik des dentschen Ritterorlens rerfaßt hat, vul. Wotschke, Herzog Albrecht und die preußischen Chroniken. Altur. Monatsschrift 49, S. 528.

1) Wie Achill Scipio unter dem 14. Oktober 1562 aus Wilna lierichtet, hatte Heraklid, nm seiue Stellung zu festigen, um die Hand der Tochter des Fiirsten Konstantin ron Ostrng geworben. "Wen sybenden September ist die walachische Fodschaft nach Kyow ankomen und ... bey meinem gnedigen Fürsten Konstantino, Herzog zn Ostrow, umb seine geliebte Tochter mith Nameu Elisabeth ansprechen lassen, das Sein Fürstliche Gnad gemelten Welachen (als Dyschpoth) nach christlicher Orduung vertraues und geben woldt." Da die begehrte Brant aber erst sicben Jahre alt war, lehnte Fürst Konstantin die Werbung ab. Heraklid bat dann die Tuchter des evangeliscben Woiwoden ron Krakaı Martin Zborowski, Christine, heimgefiibrt. $156 \pm$ warb um die junge Witwe der Hanptmann von Samogitien Johann Chodkiewiecz. Dieser hatte 1550 in Leipzig studiert, war anfänglich evangelisch, wurde aber durch Hosius (vgl. sein Schreiben vom 30. Oktober 1567, Hosii opera II. 243) für den Katholizismus gewonnen und mit ihm der Melanchthonschüler Georg Weigel aus Nürnberg, der seine Königsberger Stellung anfgegeben hatte und in des Chodkiewitz Diensteu getreten war.

2) Als Heraklid von den aufständischen Walachen unter Stephan Tomza in Suczawa belagert wurde, eilte der Kosackenfïhrer und Abenteurer Demetrins Wisniowicki herbei, nm ihn zu entsetzen. Er hatte aber kein Gliick. Erhard von Kunbeim berichtet Radom, den 24. September 1563 dem Herzog Albrecht: „Von Zeittnngen ist sider meinem, nechsten Schreiben alhier bey mir nichts sunderlichs fürgefallen, allein das viel unbeständige Redenn gehenin, wie es jetzt jnn der Wallachey so wüst und seltzan zugehe. Das letzte Geschrey aber ist das, das man sagt, der Wischnewetzky sey gewislichen gefangen vonn dem Thomze, welcheun die Wallachen für ein veueu Woywodenn anfgew orffenn. Wo aber gemelter Wioschnewetzky hinkhommeun, kann 
machte seinen verheißungsrollen reformatorischen Bemuhungen and den hohen Hoffnungen, die man in Königsberg and Wittenberg, in Zürich and Genf fïr sein Werk hegte, - im Theologischen Literaturbericht 1917 habe ich sie geschildert. - ein jähes Ende.

Von den Wittenbergern war, wie schon erwähnt, Melanchthon dem fahrenden Ritter Heraklid befrenndet. Gern batte er sich mit diesem in seinem geliebten Griechisch unterhalten. Noch näher stand ihm aber Justus Jonas der Jüngere, seit 1561 Lehrer des Kirchenrechts an der Leucorea, der Sohu des bekannten Theologen, des besten Freundes unseres Lather. Wittenberg, den 2. November 156? schreibt er dem Herzog Albrecht nach Königsberg ${ }^{1}$ ): „Ich kann

man noch nit wissen. Seine zwen Brüder sollenn jnn der Schlacht blieben sein, den übrigen Polen, so anff seiner Seitten gewesenn, sollen die Wallachen Nasen und Ohren abgeschnitten und sie wieder also jnn Polen geschickt haben zam Zaichen, würdenn hinfurt mebr die Polen wider die Wallachen etwas feindlichs füruehmen, sollten sie gleichfalls wie diese wiederumb zu den Ibrigen abgetertigt werden. Der Despot aber soll vonn dem veuen Weywoded, welcher doch khein Geschütz baben soll, zu Satzow hart belagert sein. DiB schreib ich, wie man itzt alhier danon redet. Was aber die Zeit hernach gibt, soll E. F. D. gleichfalls nnverhalten bleibenn." An Tomza nalım Rache der Woirode von Podolien Georg Jazlowiecki, 1564 Gesandter in Konstantinopel, der Schutzherr des Erangeliums in Jazlowiec südlicb von Buczacz, desseu Sübne Andreas, Petrus und Jakobus 1568 die Frankfurter Hochschule bezogen, Petrus 1563 schon die Leipziger, am 1. Oktober 1583 anch noch die Altdorfer, nach dem Tode des Vaters $(\dagger$ 1575) freilich am 27. September 1585 auch die Ingolstadter. Der Züricher Simler hat 1568 Georg Jazlowiecki neben anderen polnischen Großen sein Buch „De aeteruo dei filio Jesu Christo" gewidnet.

1) Unter dem 18. März 1563 empfahl daranf der Herzog unsern Jonas dem Fürsten Heraklid: Cum honestus ac eruditus vir doctor Justns Jonas in quibusdam negotiis in Valachiam Moldaviamque profecturus obnixe nos rogaret, at se commendatione nostra, quam pro amicitia nostra multum sibi profuturam esse confideret, ad Illtem Vram, quae eum in longinguis ac peregrinis istis regionibus gratia et favore complecteretur, prosequeremur, tanto facilius id a nobis obtinuit, quod nobis exponeret Illt Vrao in Galliis, cum ibi cum comite Mansfeldensi esset, notum fuisse eidemque, cum fortunam adversam baberet, obsequia sua animo prompto praestitisse. Illtem itaque Vram amice rogamus, ut hunc doctorem Justum benevolentia prosequi dignetur." In den Tagen, da Jonas diesen Empfehlungsbrief an Heraklid in 
E. F. D. nicht bergen, daß mein guediger ber graff Volrath von Mansfelt mich in die Walachey an den berren Despot abzufertigen willens, mir auch zu solcher reise albereit pferde vnd wagen zugestellt. Bin theglich der rolkomlichen abfertigung gewertig. Dieweil aber den herren bisweilen allerley hinderung vorfalien, so stehe ich solcher reise halben noch in Zweifel. Wo sie fort gebet, so will ich meinen weg stracks aufs landt zn Preuben zunehmen rnd ron dannen vormittels göttlicher hilffe vud E. F. G. gnedigster furderung durch land zu Polen in die gedachten lande zihen. Hoffe, es solle mir aus solcher reise grosse wohlfahrt entstehen. Dann ich hochgedachten hern Despot anch eine Zeit, als er in Frankreich zu vufahl kommen, das leben geredt vod von dannell in Deatschlandt gebolffen, do ibme dann wohlgedachter mein gnediger graf Volradt viel ehre vod freundtschaft bewiesen, des er sonder $\mathrm{Zweiffel}$ eingedenk rnd mich geniessen lassen wird. Do sichs aber zutrüge, das mich wohlgedachte graffen nicht schickten vud E. F. D. achteten es dafür, das ich solche reise obne sonderlich große eaßerste gefahr thun könute, so were mein vnterthenigste bitt, E. F. G. wollen mir die gnade erzeigen vod mich von jhrentwegen an gedachten hern Despoten abfertigen ${ }^{1}$ ). Was nuh E. F. D.

Wittenberg ausgehändigt erhielt, beschwerte sich über den Fürsten bitter Albrecht Laski bei dem preußischen Gesandten Christoph Jouas auf dem Petrikauer Reichstage. Petrikan, den 8. A pril schrieb er anch dem Herzoge Albrecht karz: „Putari non esse intermittendum, quin Ill. Dom ${ }^{n i}$ V. quid inter d. despotam, palatinatus Moldarici principem, et me agatar, planum facerem, praesertim cum caeteris christianis principibus, quibus ab illo iniariis affectus sim, indicarerim. Sed quia in praesentia literas meas seriis et gravibus Ill. Domia V. negociis nolebam esse impedimento, nec fuit admodum ea mihi valetudo, ut id praestare possem, d. Christophoro Jonae, Ill. Domuis V. consiliario, abande enarrata commisi, ut Ill. Dom ni $\nabla$. meo nomine referret."

1) Vom 12. Dezember 1562 ist die Antwort des Herzogs datiert. „Was die reyse jnn die Wallachey betrifft, heren wir gerne, daß euch graff Volradt von Mansfeldt derer ort abzufertigen willens. Dieweyll jhr aber doch noch in zweiffel stehet, ob solche reyse jren fortgang gewinne oder nicht vndt $\nabla f f$ dell fall, das gedachter graffe anders sinnes würde, bittet, wir euch die gnade erzeygen vndt vor vos hinein schicken wollten, seint wir jn gnaden zufrieden, wo gemelter graffe euch nicht schickte, das jhr euch nichts weniger za vns hereyn begebet, so wollen wir euch jn vaserm namen, dieweyll jhr dadurch auch vermeint curen nutz zu schaften, den wir jnn gnaden gerne ferdern, dahin zum Despota abfertigen, vndt kann solche reyse ohne gefhar vollendet werden. Der graff schicke ench nhun oder nicht, so wollet es also machen, daß jhr von gedachtem grauen werbung 
disfals jn gnaden ror guth ansebn, bit ich rnterthenigst, E. F. D. wolten mir bey diesem bothen zuschreiben lassen, disse dinge aber jn gebeim zu halten gnedigst befehlen. schicket mich der graf, wie ich boffe, so will ich, ob gott will, jnn kurz selbst bei E. F. D. sein."

Zwei Monate später, am 10. Januar 1563, laibt sicb Justus Jonas dem Herzog gegenuher vermehmen: „Dit reise jn die Wallachey zu dem herm Despot geht für sich. Nachtem sichs aber vieleicht noch ein wochen oder etlich verzieben möchte, als bitt ich E. F. D. auffs voterthenigst, sie wollen nir auffs erst, als es immer möglich sein kam, jn gnaden rermelden, ob jeh auch bequemlich vid sicher ron Konisperg aus der orthe kommen müchte. Denn etliche meinen, es sey durch Siebenblirgen vind an die Sichlesien der negste und beste weg. Etliche meinen aber, der wegdurch laud zu Polen sey der sicherste ${ }^{1}$ ). Icb bitt aufs denuitigst rnd jn vnterthenigkeit, E. F. D. wollen mir disfalls jr gnedigst bedenken vermelden lassen ${ }^{2}$ )." Noch im fol.

ahn rns auch haben möget oder each dermaßen stellen, als ob jhr ettwas ron ihme oder andern ahn pns zu bringen bettet. Jue ehe jhr ench nhnn hereyn begebet, jhe besser solches ist. Wollet euch derhalben selbst nicht säumen."

1) Unter dem 7. Febraar 1063 autwortete der Herzog: ., so viel eure wallachische reise belanget, könuet jr dieselbige vasers erachtens am fïglichsten rnd sichersten durch Preussen thun vod rortstellen, darzu wir ench dann zu enr anknuft fernere anleytmug geben wollen. ... Was aber ener bitten der bewußten summa halben, damit jr rns verhafft, belanget. das wir rff den fall, jr anff ter wallachischen reine bleiben rnd mit lode abgehen soltet, eurem weibe alsdann die helffte derselbigen erlasseu wollten, wollen wir zu gott hoffen, der werde auf dieser reisen, wie zuuorn aufl anderen mehr geschchen, mit seinen heiligeu engel $n$ bei euch sein."

') Am 19. Järz 1563 ließ ihm Herzog Albrecht in Beantwortung von drei weiteren Briefen schreibcn: "Wir hälten gerno geseben, daß colche reyse lengst jren vortyang gehapt, weil es aber nicht greschehen können, stellen wir solches nunmebr auch an seinen orth. Wir weren aber noch woll wie znuorn geneigt, euch vor vnsere person an denselbigen orth zu schicken, so ist es aber an dem, das der herr despot neulicher zeitt seine bottschaft bei vns gehapt, mit welcher wir uunmehr rnsere sacheu, so wir bei s. l. auszurichten, rortgestellet, daß wir dismal nichts bei s. l. zn thun haben und euch also jtziger \%eitt der mühe vberheben vnd die rnkosten sparen können. Beilanken rns aber eures vnterthenigen geneigten gutten willens vud schicken euch gleichwol vff den fall, das euch der von Jansfelt noch schicken wiirde, eurem bitten nach die vorschrifften an gedachten berrn Despoten. 
uenden Mai truy sich Junas mit lieiseplanen. Am 9. dieses Ionats schreibt er dem Herzog aus Leipzig: . Mein gnediger graf Volrath hat mich anher gen Leipmig beschieden in meinung, mich in kurz in die Walacbey ah\%ufertigen. Hotie bei seinan gnaden $z$ u erhalten. $d a b$ ich meinen weg auf f. F. D. lande zu nebmen möge, als will ich (tliche gelegenheit vermelden, darob sie sicb verwundern werden." Eine latırivierige schmerzliche Krankheit, verbunden mit Kopfgicht, zwang Jonas, seine Reise weiter hinauszuschieben. Daun liefen Nachrichten rou Heraklids böser Lage, schlieblich von seinem Tode ein und machten sie unnötig.

$\mathrm{N}$ a c h trag. Einen Brief des Heraklid an Melanchtbon vom 23. November 1556 aus Brüssel bietet Crusius, Turcogräcia.

Anscheinend noch beror Heraklid za Albrecht Laski kam, sandte dieser unter dem 13. November 1559 seiven Sekretair Erasmas Krossenski zar Ordination nach Wittenberg. Einen Brief an Melanchthon gab er ihm mit. Archiv VI, S. 354. Als letzter des Jahres 1559 erhielt Krossenski die Amtsweihe in der Elbstadt. Buchwald, Wittenberger Ordiniertenbuch $1 \mathrm{Nr}$. 1943. Für "vociert ad d. albertum anascam in Kesmarch" ist hier naturlich ad d. Albertam Alaseum zu lesen. Das Ordinationszengnis Corp. Reform. IX Nr. 6890. Jener Johann Sommer, der im September 1562 zu Lublin in Heraklids Dienste getreten war, an der Besitznalıme Chotins im Januar 1563 teilgenommen hatte, dann der Bibliothekar Heraklids, auch Lehrer an der von ibm gegründeten evangelischen Lateiuschule wurde und glucklicher als der evangelische Bischof der Moldau, Johann Lusenski, Calvins "gelieber Bruder", der bei dem Aufstande der Walachen vergiftet, dessen Fran gebenkt wurde, sein Leben za retten waßte, ist nicht ein Sohn des Käsmarker Pfarrers Johann Sommer, der am 22. Februar 1548 in Wittenberg von Bugenhagen die Ordination empfangen" hat. Er stammte aus Pirna und hat sich noch Fribjahr 1562 in Frankfurt einschreiben lassen. Es ist der bekannte Antitrinitarier, der Clausenbarger Rektor. Wir verdanken ihm eine Lebensbeschreibang Heraklids. Vgl. Legrand, Deux vies de Jacques Basilicos.' 'Paris 1884. 